\title{
Gas Breakdown Limits For Inverse Cherenkov Laser Accelerators *
}

\author{
Y.Liu \\ Physics Department of UCLA \\ Los Angeles, CA 90024 \\ I.V.Pogorelsky \\ Brookhaven National Laboratory \\ Upton, NY 11973
}

\begin{abstract}
The probability of avalanche, tunneling and multiphoton ionization induced by a $\mathrm{CO}_{2}$ laser in $\mathrm{H}_{2}$ gas has been calculated. Laser light screening by a self-induced plasma density gradient is considered as the limiting factor for upscaling a $\mathrm{CO}_{2}$ laser-driven Inverse Cherenkov Laser Accelerator beyond $650 \mathrm{MeV} / \mathrm{m}$. However, in near-resonace inverse Cherenkov acceleration where a shorter wavelength laser is used at a wavelength near the resonance of the gas (e.g. $248 \mathrm{~nm}$ in $\mathrm{H}_{2}$ ), the formation of a plasma is not a problem because the plasma density is below the critical density. In that case, the laser beam propagates unaffected through the plasma and the acceleration gradient is not limited by gas breakdown. Gradients $>1 \mathrm{GeV} / \mathrm{m}$ are possible.
\end{abstract}

\section{Introduction}

With contemporary high-peak-power picosecond lasers, extremely high intensity levels, $\geq 10^{18} \mathrm{~W} / \mathrm{cm}^{2}$, are attainable upon tight focusing of laser beams. The transverse electric field corresponding to such an intensity is about $30 \mathrm{GV} / \mathrm{cm}$. The idea to use at least a portion of this enormous field for particle acceleration is promising. Several laser accelerator schemes are presently under development worldwide. One of them is the Inverse Cherenkov Accelerator (ICA) ${ }^{1}$ illustrated by Fig.1. It is based upon axicon focusing of a radially-polarized laser beam onto the electron beam axis at the phase matching angle $\theta_{c}$ that satisfies the Cherenkov condition

$$
n \beta \cos \theta_{c}=1,
$$

where $n$ is the refraction index of the medium and $\beta$ is the normalized electron velocity. In a recent experiment at the Brookhaven Accelerator Test Facility (ATF), up to $3.7 \mathrm{MeV}$ acceleration over a $12 \mathrm{~cm}$ interaction length in $2 \mathrm{~atm}$ of hydrogen gas was demonstrated with $\mathrm{a} \sim 580 \mathrm{MW} \mathrm{CO}_{2}$ laser pulse at $\theta_{c}=20 \mathrm{mrad}$. Since the acceleration effect is scalable proportional to the square root of the laser power, $\sim 0.5 \mathrm{GeV} / \mathrm{m}$ acceleration is predicted if a $1 T W$ laser is used. Such acceleration gradientesare attractive foefdeveloping eompact ...- 
$1 \mathrm{GeV}$ electron accelerators for industrial and medical applications, as well as for projected next generation laser linacs reaching the $1 \mathrm{TeV}$ energy level. Research in this direction is under way. A $1 T \mathrm{WCO}_{2}$ laser is under development at the $\mathrm{ATF}^{3}$ and a $100 \mathrm{MeV}$ ICA demonstration experiment is starting that will eventually lead to $1 \mathrm{GeV}$ proof of principle ICA experiments. ${ }^{4}$

However, a possible limitation for increasing the accelerating field in ICA may be optical breakdown of the gas medium needed to maintain the phase matching condition, Eq.(1). The breakdown plasma will refract and reflect laser radiation, which reduces the accelerating electrical field acting on the electron beam.

We address here the problem of gas breakdown under the conditions of the ICA experiment at the ATF. Several laser-induced gas breakdown mechanisms, such as tunneling, multiphoton and cascade ionization will be discussed in order to estimate the ultimate acceleration gradient attainable with the ICA technique prior to gas breakdown. We show that $0.6 \mathrm{GW} / \mathrm{m}$ is the maximum peak acceleration that may be attained with the $\mathrm{CO}_{2}$ laser driver. In Section 2, the threshold electron density gradient which can screen the $e$-beam from the laser driver is estimated. In Section 3, various mechanisms of plasma buildup are analyzed. The ultimate acceleration gradient for the conditions of the ATF ICA experiment is estimated in Section 4. In Section 5, the advantages of low gas pressure and a short-wavelength laser driver for prospective ultra-high gradient ICA are briefly discussed. Under these conditions gas breakdown is not an issue because the plasma does not disrupt the laser beam.

\section{Reflection of Laser Radiation by Plasma}

When laser light propagates through an overdense plasma with an electron density $N_{e}$ above the critical plasma density $N_{c r}$, the laser beam is strongly attenuated due to the resonant absorption by laser-induced plasma waves. ${ }^{5}$ The critical density $N_{c r}$ is determined by the equation

$$
N_{c r}=\frac{\pi m c^{2}}{e^{2} \lambda^{2}}
$$

where $e$ and $m$ are the charge and mass of the electron, respectively, and $c$ is the speed of light. We see that $N_{c r}$ scales proportionally to $\omega^{2}$ (or $\lambda^{-2}$ ), and $N_{c r}=1.0 \times 10^{19} \mathrm{~cm}^{-3}$ for $\mathrm{CO}_{2}$ laser radiation $(\lambda=10.6 \mu \mathrm{m})$. For the ICA experiment, we are also concerned with the early stage of underdense plasma formation. Under oblique incidence of the laser beam onto a plasma layer, which is the case for the ICA geometry, refraction and reflection of the laser beam may result in partial or complete screening of laser radiation. This situation will occur at plasma densities $N_{e}$ much below $N_{c r}$.

Refraction of laser radiation on a plasma layer results from changes of the gas refraction index $\Delta n$ according to the equation

$$
\frac{\Delta n}{n} \approx-\frac{N_{e}}{2 N_{c r}}
$$




\section{DISCLAIMER}

Portions of this document may be illegible in electronic image products. Images are produced from the best available original document. 
which follows from the plasma refraction law $n=n_{0} \sqrt{1-\left(N_{e} / N_{c r}\right)^{2}}$. For an electromagnetic wave incident onto a plasma layer at angle $\theta_{c}$, the reflection condition is

$$
|\Delta n| \geq n\left(1-\cos \theta_{c}\right)
$$

or for oblique incidence

$$
|\Delta n| \geq \frac{1}{2} n \theta_{c}^{2}
$$

and by Eq.(3)

$$
N_{e} \geq N_{c r} \theta_{c}^{2} \text {. }
$$

With $\theta_{c}=20 \mathrm{mrad}$, the total reflection of the axicon-focused laser beam may be observed at a plasma density as low as $N_{e}=4 \times 10^{-4} N_{c r}$. Hence, for the $\mathrm{CO}_{2}$ laser-driven ICA experiment at the ATF, the threshold plasma density is $N_{e} \approx 10^{16} \mathrm{~cm}^{-3}$.

\section{Laser-Induced Gas Breakdown Mechanisms}

At the extremely high laser intensities attainable with picosecond pulses, tunneling and multiphoton ionization are usually the main mechanisms of gas breakdown. The intensity threshold $I_{t h}$ for these processes depends on the laser wavelength $\lambda$ and the ionization potential $U_{i}$ of the medium. The magnitude of $I_{t h}$ is not as sensitive to the laser pulse duration $\tau_{p}$ and does not strongly depend upon the gas pressure. Because the ionization potential of any atom is very much higher than the photon energy of $10 \mu \mathrm{m}$ light (i.e., $0.1 \mathrm{eV}$ ), multiphoton ionization is least likely to occur with $\mathrm{CO}_{2}$ laser light(see Appendix A), and the tunneling process is prevalent during strong irradiation conditions.

Another mechanism that causes gas breakdown is cascade (avalanche) ionization, where a free electron acquires energy from the laser electromagnetic wave via elastic collisions with neutral atoms. After several collisions it may obtain enough energy to ionize the atom and create second-generation free electrons. Depending upon the laser pulse duration and intensity, this process can continue until gas breakdown occurs. Hence in addition to $\lambda$ and $U_{i}$, gas pressure and $\tau_{p}$ are the major factors in determining the laser intensity threshold for avalanche breakdown.

\subsection{Tunneling.Ionization Probability}

The tunneling and multiphoton ionization probabilities are both described by one general Keldysh formula, ${ }^{6}$ but under different conditions for the parameter

$$
\gamma=\frac{\omega \sqrt{2 m U_{i}}}{e E}
$$

where $E$ is the laser electric field strength. For $\gamma \gg 1$ the ionization can be described as a multiphoton process through which the valence electron can escape from the atom by simultaneously absorbing several photons. This process generally prevails in the relatively 


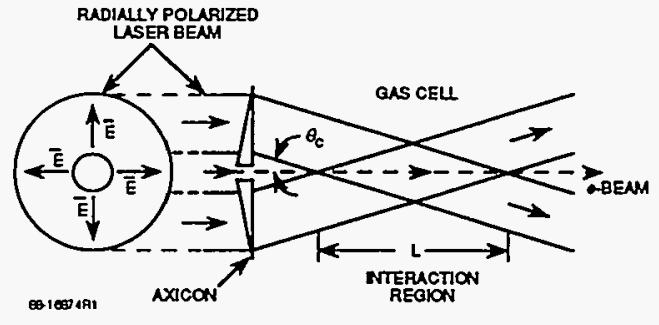

Figure 1: Axicon focusing geometry.

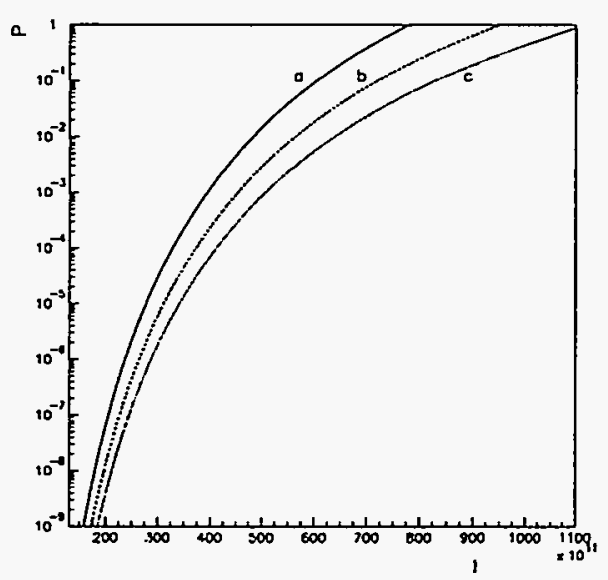

Figure 2: The tunneling ionization probability $P$ vs laser peak power $I\left[\mathrm{~W} / \mathrm{cm}^{2}\right]:$ a) pulse width $\tau_{p}=50 \mathrm{ps}$ and integration time $\Delta t=50 \mathrm{ps} ; \mathrm{b}$ ) $\tau_{p}=\Delta t=10 p s ;$ c) $\tau_{p}=\Delta t=3 p s$.

"weak"-field, high-frequency regime. For $\gamma \ll 1$ the ionization can be described as a tunneling process which happens in the strong-field, low-frequency limit. The physical sense of the condition $\gamma=1$ is the equality of the pondermotive potential of the free electron oscillating in the laser field to the ionization potential of the medium.

Under the $\gamma \ll 1$ limit (laser pulse intensity $I \gg 7.4 \times 10^{11} \mathrm{~W} / \mathrm{cm}^{2}$ for $\mathrm{CO}_{2}$ laser in $\mathrm{H}_{2}$ gas) the formula for the tunneling ionization rate $A$ becomes ${ }^{6,7}$

$$
A=13\left(\frac{(6 \pi)^{6} m U_{i}^{7}}{e^{2} E^{2} h^{6}}\right)^{1 / 4} \exp \left[-\frac{1.5 \pi \sqrt{2 m U_{i}^{3}}}{e E h}\left(1-\frac{m \omega^{2} U_{i}}{5 e^{2} E^{2}}\right)\right] .
$$

where $U_{i}=15.4 \mathrm{eV}$ for $H_{2}$ and $h$ is Plank's constant. Using

$$
I=\frac{1}{2} \epsilon_{0} c|E|^{2}
$$

where $\epsilon_{0}$ is the electric permittivity in vaccum, then Eq. (6) becomes

$$
A \approx 2.7 \times 10^{6} \times I^{-1 / 4} \exp \left(-1.5 \times 10^{2} \times I^{-1 / 2}\right),
$$

where $A$ is in $p \sec ^{-1}$ and $I$ is in $T W / \mathrm{cm}^{2}$.

To find the ionization probability function $P\left(I_{0}\right)$ behavior versus laser intensity, we integrate $A(t)$ over the laser pulse duration $\Delta t$ with $t=0$ corresponding to the peak of the 
pulse

$$
P\left(I_{0}\right)=\int_{-\Delta t / 2}^{\Delta t / 2} A(t) d t
$$

The computed plots of $P\left(I_{0}\right)$ for a Gaussian laser pulse with the intensity distribution expressed as

$$
I(t)=I_{0} \exp \left[-\left(\frac{2 t}{\tau_{p}}\right)^{2}\right]
$$

are presented in Fig.2. Curve a corresponds to the pulse width $\tau_{p}=50 p s$ and the integration time $\Delta t=50 p s ;$ curve $\mathbf{b}$ is for $\tau_{p}=\Delta t=10 p s$; and curve $\mathbf{c}$ is for $\tau_{p}=\Delta t=3 p s$. Note, that an increase of the integration time to $\Delta t=2 \tau_{p}$ does not change the results shown in Fig.2. This indicates that only the peak field is important in the tunneling process.

As discussed in Section 2, the local gas breakdown limit can be set to $N_{e} / N_{c r}=4 \times 10^{-4}$. For the $1.7 \mathrm{~atm}$ of hydrogen used in the ATF ICA experiment, $N_{m} / N_{c r} \approx 4.5$, where $N_{m}$ is the number of molecules per $\mathrm{cm}^{3}$. Hence, we take $P=2 \times 10^{-4}$ as the criteria for gas breakdown. Then, the threshold laser intensity is $I_{t / 2}=32 T W / \mathrm{cm}^{2}$ for $\tau_{p}=50 \mathrm{ps}$, and $I_{t h}=42 T W / \mathrm{cm}^{2}$ for $\tau_{p}=3 p s$.

\subsection{Gas Breakdown by Avalanche}

The avalanche in the light-wave electrical field is another mechanism that can cause gas breakdown. ${ }^{7,8}$ To initiate the avalanche, the existence of primary free electrons is necessary. Assume that the primary free electrons are produced from impurities within the gas with lower ionization potential or via tunneling ionization as discussed previously. Those free electrons exposed to the laser's electromagnetic field will oscillate with the laser frequency. The energy within such an oscillator, called the pondermotive potential, is equal to

$$
\varepsilon_{o s c}=\frac{e^{2} E^{2}}{2 m \omega^{2}}
$$

Energy accumulation by the electron is possible via elastic collisions with neutral molecules, in which the energy of the oscillating motion is transformed into the kinetic energy of longitudinal motion of the electron. The average electron kinetic energy increment with each collision is $\varepsilon_{o s c} / 2$, and the growth rate of the electron longitudinal kinetic energy $\varepsilon$ under the influence of the electromagnetic wave is

$$
\frac{d \varepsilon}{d t}=\left(\frac{e^{2} E^{2}}{4 m \omega^{2}}\right) \nu_{e f f}
$$

where $\nu_{e f f}$ is the effective electron-atom momentum transfer collision frequency. By definition $\nu_{e f f}=N_{m} v \sigma$, where $v$ is the electron velocity and $\sigma$ is the elastic scattering crosssection. Note that $\nu_{\text {eff }}$ is not a sensitive function of $\varepsilon$ and may be considered as a constant in the course of these calculations. ${ }^{9,10}$ Referring to Eq.(7), we obtain

$$
\frac{d \varepsilon}{d t}=\left(\frac{e^{2}}{2 c \epsilon_{0} m \omega^{2}}\right) I \nu_{e f f}
$$


We assume that electrons loose their energy primarily via ionization of the $\mathrm{H}_{2}$ molecules. The recombination and diffusion electron losses are considered negligible. In order to ionize $H_{2}$ molecules, the free electron kinetic energy must reach a value of $\varepsilon=2 U_{0}+U_{i}$, where $U_{0}$ is the initial secondary electron mean energy. Disregarding other electron energy losses except for ionization, energy conservation requires that

$$
\left(U_{0}+U_{i}\right) \frac{d N_{e}}{d t}=N_{e} \frac{d \varepsilon}{d t} .
$$

After integrating Eq.(14) over the laser pulse duration, using Eq.(13) and assuming a Gaussian pulse shape [Eq.(10)], we obtain

$$
I_{0}=\frac{4 \epsilon_{0} c\left(U_{0}+U_{i}\right) m \omega^{2} \ln \left[N_{e}\left(\tau_{p}\right) / N_{e}(0)\right]}{\sqrt{\pi} \tau_{p} e^{2} \nu_{e f f}} .
$$

Setting $N_{e}\left(\tau_{p}\right) / N_{e}(0) \approx 10^{14}$ as the condition for gas breakdown ${ }^{7}$, and $U_{0} \approx U_{i}$ according to momentum conservation with angular averaging ${ }^{6}$, the gas breakdown threshold intensity is given by

$$
I_{t h}=\frac{140 \epsilon_{0} c U_{i} m \omega^{2}}{\tau_{p} e^{2} \nu_{e f f}}
$$

So far, we have not considered inelastic collisions other than ionization. An appreciable proportion of the laser energy is distributed over molecular vibrational and rotational levels. For our simplified estimates, we can use empirical observations that inelastic losses result in $2-3$ times increase of the threshold intensity above that calculated by Eq.(16). ${ }^{6}$ Ultimately, taking $v \sigma \approx 1.6 \times 10^{-7} \mathrm{~cm}^{3} \mathrm{~s}^{-1}$ for $\mathrm{H}_{2},{ }^{9,10} \nu_{\text {eff }}=7.5 \times 10^{12} \mathrm{~Hz}$ at $1.7 \mathrm{~atm}$ of hydrogen, the intensity threshold for avalanche breakdown produced by a $\mathrm{CO}_{2}$ laser pulse simply follows the relationship

$$
I_{t h} \approx \frac{30}{\tau_{p}}
$$

where $\tau_{p}$ is in $p s e c$, and $I_{t h}$ is the peak power in $T W / \mathrm{cm}^{2}$.

\section{Specifics of Axicon Focusing}

When a radially polarized beam is focused by an axicon, a circularly symmetric interference pattern is developed along the optical axis of the axicon. The analytical solution for the radial component of the electrical field $E_{r}(r, z)$ is a Bessel function of the first kind of order 1:

$$
E_{r}(r, z)=E_{0}(z) \times J_{1}\left(2 \pi \theta_{c} r / \lambda\right)
$$

where $E_{0}$ is the field amplitude depending upon the input laser intensity distribution at the axicon surface $W(R)$ (we introduce the new symbol $W$ to avoid confusion with the laser intensity $I$ at the axicon focus, $R=z \theta_{c}$ ),

$$
E_{0}(z)=2 \pi \theta_{c} \sqrt{\frac{2 z W(R)}{\epsilon_{0} c \lambda}}
$$


The first maximum of the radial distribution $E_{r}(r, z)$ is at

$$
r_{m a x}=\frac{0.29 \lambda}{\theta_{c}}
$$

and is equal to $170 \mu \mathrm{m}$ for $\lambda=10.6 \mu \mathrm{m}$ and $\theta_{c}=20 \mathrm{mrad}$. A plot of the $E_{r}(r)$ distribution is presented in Fig.3a.

(a)



(b)



Figure 3: Electrical field distribution of axicon-focused radially polarized laser beam. a) radial component $\left.E_{r}(r) ; \mathrm{b}\right)$ longitudinal component $E_{z}(r)$.

Unlike the radial component, the longitudinal component produced by the axicon conical wave front can be expressed analytically by a Bessel function of the first kind of order 0 and has a maximum along the optical axis: ${ }^{1}$

$$
E_{z}(r, z)=\tan \left(\theta_{c}\right) \times E_{0}(z) \times J_{0}\left(2 \pi \theta_{c} r / \lambda\right) .
$$

The amplitude of this component defines the peak ICA acceleration gradient attainable under phase-matching condition Eq.(1).

Note that for $\lambda=10.6 \mu \mathrm{m}$ and $\theta_{c}=20 \mathrm{mrad}$ the radial position of the first minimum in the distribution given by Eq.(21) is at

$$
r_{\min }=\frac{0.38 \lambda}{\theta_{c}} \approx 200 \mu m .
$$

Hence, the first minima and maxima in the $E_{r}(r)$ and $E_{z}(r)$ distributions nearly coincide with each other. By using Eqs.(19)-(21), the ratio of the first maxima of the $E_{r}(r)$ and $E_{z}(r)$ distributions is

$$
\frac{E_{r}^{\max }}{E_{z}^{\max }}=\frac{J_{1}(0.58 \pi)}{\tan \left(\theta_{c}\right) J_{0}(0)} \approx 29,
$$


for $\theta_{c}=20 \mathrm{mrad}$. This implies that the $E_{\mathrm{r}}$ field should be primarily responsible for gas breakdown development around the $e$-beam axis and the peak acceleration is intimately related to the previously defined ionization threshold via the $E_{r} / E_{z}$ ratio. According to Eq.(23) and Eq.(7) we can estimate the maximum attainable acceleration gradient $E_{z}^{\max }$ along the $e$-beam axis before gas breakdown at the maximum of the $E_{r}(r, z)$ distribution

$$
E_{z}^{\max }=\theta_{c} \frac{J_{0}(0)}{J_{1}(0.58 \pi)} \sqrt{\frac{2 I_{t h}}{\epsilon_{0} c}} .
$$

If $E_{z}^{m a x}$ is in $M V / c m$ and $I_{t h}$ is in $T W / \mathrm{cm}^{2}, E_{z}^{m a x} \approx \sqrt{I_{t h}}$.

Combining Eq.(24) with Eq.(17) for avalanche ionization in $\mathrm{H}_{2}$ gas, we estimate the peak acceleration gradient which may be attained in the ICA experiment depends upon the $\mathrm{CO}_{2}$ laser pulse width $\tau_{p}$. For example, for a bandwidth-limited $3 p s \mathrm{CO}_{2}$ laser pulse, ${ }^{11}$ the maximum predicted acceleration gradient is $E_{z}^{\max } \approx 320 \mathrm{MeV} / \mathrm{m}$.

However, there is a possibility for further increase of $I_{t h}$ by enhancing inelastic electron energy losses by using a quenching gas diluted in hydrogen (for example, Argon). In view of this consideration, it looks more appropriate to invoke the tunneling ionization threshold when determining the absolute limit for $\mathrm{CO}_{2}$ laser-driven ICA acceleration. Using $I_{t h}$ data for the tunneling ionization obtained in Section 3.1, we have $E_{z}^{\max } \approx 650 \mathrm{MV} / \mathrm{m}$ as the maximum feasible acceleration gradient for the ICA scheme under the ATF experimental conditions.

In addition optical damage of the axicon mirror may be another factor to limit the input laser intensity. According to available experimental results on optical damage with picosecond $\mathrm{CO}_{2}$ laser pulses, $5 \mathrm{~J} / \mathrm{cm}^{2}$ fluence is approximately the damage threshold for a copper axicon mirror. ${ }^{12}$ Using Eq.(18), Eq.(19) and the relation $R=z \theta_{c}$, we derive a condition for the safe delivery of the threshold intensity to the interaction region:

$$
R \times W_{d}>\frac{\lambda}{\left(2 \pi J_{1}(0.58 \pi)\right)^{2} \theta_{c}} I_{t h}=4.0 \times 10^{-3} I_{t h},
$$

where $W_{d}$ is the damage intensity defined as the damage fluence divided by $\tau_{p}, \lambda$ is $10.6 \mu \mathrm{m}$, $\theta_{c}$ is $20 \mathrm{mrad}$ and $R$ is in $\mathrm{cm}$. For example, in order to reach $600 \mathrm{MV} / \mathrm{m}$ acceleration gradient (corresponding to $I_{t h}=42 T \mathrm{~W} / \mathrm{cm}^{2}$ ) for the conditions of the ATF ICA experiment with $\tau_{p}=3 p s$ and $W_{d}=1.7 T W / \mathrm{cm}^{2}, R_{\min }=1 \mathrm{~mm}$.

So far, we have discussed the local peak acceleration gradients feasible for the IR ICA method and possible optical damage limitations. To estimate the bulk acceleration effect, the longitudinal distribution of the acceleration field along the $\mathrm{z}$-axis should also be considered.

Neglecting diffraction effects, the longitudinal distribution $I_{0}(z)$ for the input Gaussian laser beam described by the function $W(R)=W_{0} \exp \left[-(2 R / \Delta)^{2}\right]$ with $\Delta=5 \mathrm{~mm}$ (Fig.4a) 
is shown in Fig.4d. In a practical setup, the axicon will have a hole in the middle to introduce the electron beam to the interaction region. To avoid losing most of laser beam energy, the intensity distribution of the delivered laser beam at the axicon surface is converted into a "donut-shape" using a separate axicon telescope. The corresponding radial distribution obtained after the axicon telescope is presented in Fig.4b. Axicon focusing results in an axial distribution similar to (4d), but shifted along the $\mathrm{z}$-axis by the distance equal to the radial expansion of the axicon telescope divided by $\theta_{c}$. To achieve a constant field amplitude along the z-axis, one should ideally tailor the radial field distribution in a way presented in Fig.4c.
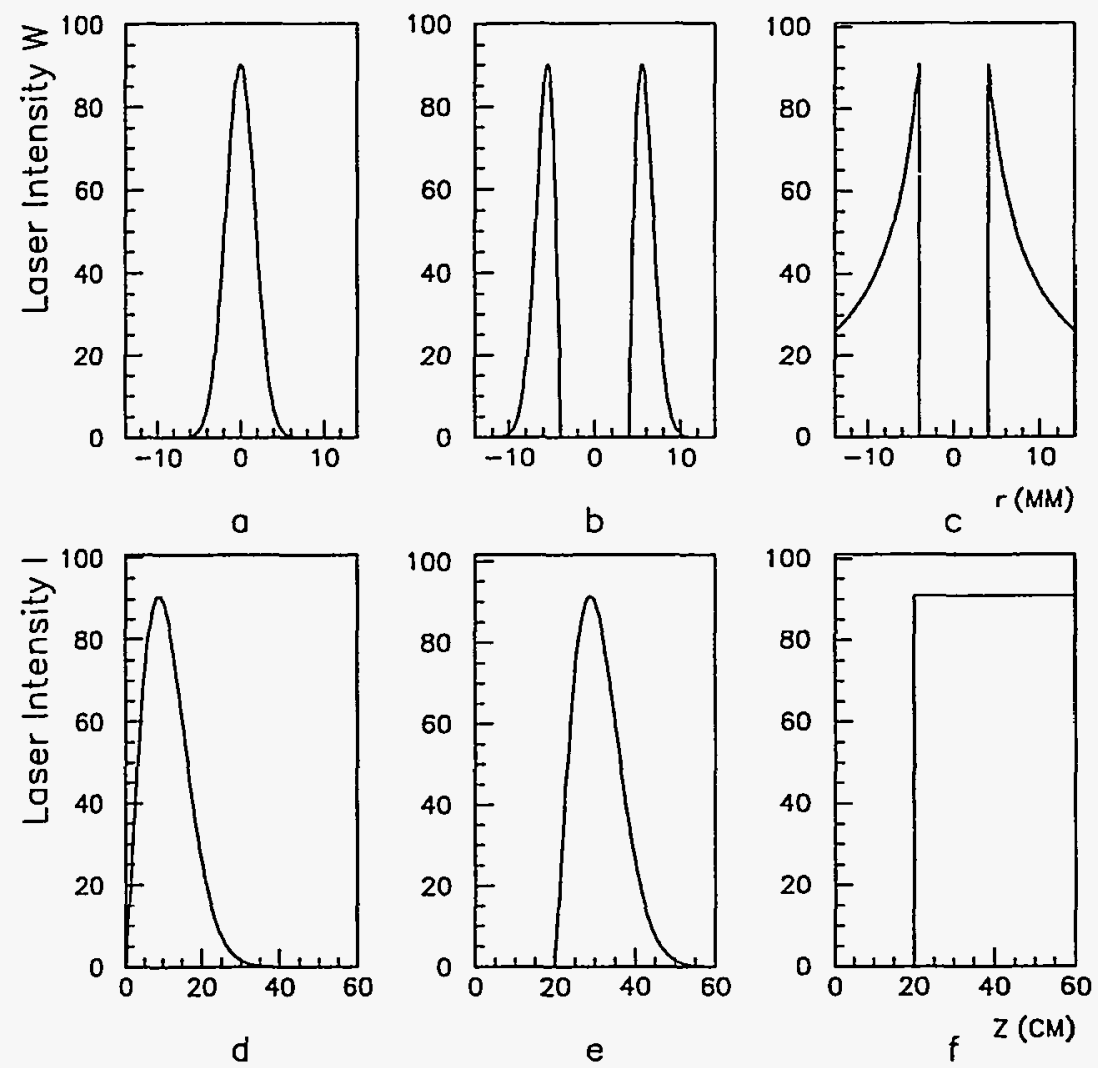

Figure 4: Initial laser beam intensity profiles $W(R)$ with corresponding distributions along the axicon focus axis $I(z)=z \theta_{a} W\left(z \theta_{c}\right)$ for $\theta_{c}=$ 20 mrad shown below: a) Gaussian beam, b) Gaussian beam axicontelescoped with radial expansion of $4.0 \mathrm{~mm}, \mathrm{c}$ ) axicon-telescoped hyperbolic beam $(W(R) \sim 1 / R)$.

As an example, we can estimate the bulk acceleration that may be obtained with a technically feasible $1 \mathrm{TW} 3 p s \mathrm{CO}_{2}$ laser beam properly distributed on the axicon to avoid surface 
damage and gas breakdown. The total acceleration length is $L=0.5 \mathrm{~m}$ if the illuminated surface over the axicon is between $1<R[\mathrm{~mm}]<11$. The theoretical bulk acceleration, defined as the product $L \times E_{z}^{\max }$, is $300 \mathrm{MeV}$. As long as a small percentage of the laser power is consumed by the accelerated electrons ( $10 \%$ for $300 \mathrm{~A}, 3 p s$ electron bunch), the spent laser beam may be recycled over several acceleration stages. (This calculation ignores the effects of gas scattering of the electrons.)

Finally, we should point out that all estimates have been done so far for $1.7 \mathrm{~atm}$ of hydrogen and a fixed Cherenkov angle of $20 \mathrm{mrad}$, which are needed to maintain phase synchronization of the laser radiation with electrons of $50 \mathrm{MeV}$ initial energy. Let us next address the question: is there the possibility of higher acceleration gradients for ICA operating with higher initial electron energies? For electron energies above $100 \mathrm{MeV}, \beta \approx 1-1 / 2 \gamma^{2}$ quickly approaches 1 and Eq.(1) may be rewritten in the form

$$
n-1 \approx \frac{\theta_{c}^{2}}{2}
$$

The increase of the axicon angle will result in a higher proportion of the laser field available for longitudinal electron acceleration. However, as required by Eq.(26), an increase in $n-1$, which is proportional to the gas pressure, results in a proportional drop of the threshold avalanche ionization intensity. Ultimately, an adjustment of the ICA parameters does not help increase the peak acceleration gradient above the previously estimated $650 \mathrm{MV} / \mathrm{m}$ value.

\section{Near-Resonant ICA With Short-Wavelength Laser Drivers}

The estimated peak acceleration gradient is about $650 \mathrm{MeV} / \mathrm{m}$ under the gas breakdown limit for the current ATF ICA experimental setup which uses a $\mathrm{CO}_{2}$ laser. This figure is high enough for planned $100 \mathrm{MeV}$ demonstration experiment and for compact $1 \mathrm{GeV}$ accelerator designs. We would like to also mention the prospects towards achieving even higher acceleration gradients by using a short-wavelength laser driver.

At a higher initial electron energy, the reduction in geometrical $e$-beam emittance and gas scattering makes it possible to achieve a tightly focused $e$-beam. It may be practical to use a shorter wavelength laser driver which is characterized by narrower axicon focusing proportional to $\lambda$ (see Eq.(18)-(22)). The advantages of shorter wavelengths are higher $N_{c r} \sim \omega^{2}$ and a lower avalanche ionization rate, because the avalanche ionization threshold is proportional to $\omega^{2}$. For shorter wavelengths, multiphoton ionization may dominate the ionization process for $\lambda \leq 1 \mu \mathrm{m}$ and give a lower intensity threshold. However, a further advantage of shorter wavelengths is the possibility of using near-resonance ICA and much lower gas pressure. ${ }^{13}$ It is possible to get $N_{m} Z \ll 10^{-3} N_{c r}$ (where $Z$ is total molecular nuclear charge) at lower gas densities, which means that, even for complete ionization, reflection of the laser beam will not occur. For example, using a $K r F$ laser driver, the hydrogen pressure can be reduced up to $0.01 \mathrm{~atm}$ and the maximum plasma density is $5.6 \times 10^{17} \mathrm{~cm}^{-3}$ while $N_{c r}=1.8 \times 10^{22} \mathrm{~cm}^{-3}$ (see Appendix B). Hence, gas breakdown would not be a limitation for scaling up the acceleration gradient, and gradients $>1 \mathrm{GeV} / \mathrm{m}$ are possible ${ }^{13}$. 


\section{Conclusions}

The gas breakdown threshold for the ATF ICA experiment is limited by laser-induced avalanche. The threshold limit will be $\sim 10 \mathrm{TW} / \mathrm{cm}^{2}$ with $3 p s$ pulse width. The maximum acceleration gradient is about $320 \mathrm{MeV} / \mathrm{m}$ for the current ICA setup. With an optimized laser intensity distribution on the axicon mirror, the maximum acceleration gradient can be $650 \mathrm{MeV} / \mathrm{m}$. We show that an adjustment of the ICA parameters does not help increase the acceleration gradient beyond $650 \mathrm{MeV} / \mathrm{m}$. But for a shorter wavelength, gas breakdown may not be a limitation for scaling up the acceleration gradient. This implies that the acceleration gradient can be beyond the $650 \mathrm{MeV} / \mathrm{m}$ limit for short wavelengths.

\section{Appendix}

\section{A. Multiphoton Ionization Probability}

In the limiting case of $\gamma \gg 1\left(I \ll 7.4 \times 10^{11} \mathrm{~W} / \mathrm{cm}^{2}\right.$ for $\mathrm{CO}_{2}$ laser in $\mathrm{H}_{2}$ gas $)$, the formula describing the multiphoton effect is ${ }^{6,7}$

$$
A=\omega n^{3 / 2}\left(\frac{e^{2} E^{2}}{8 m U_{i} \omega^{2}}\right)^{n}
$$

where $n$ is the number of quanta necessary to knock out the electron, and is equal to the integer part of the quantity

$$
n=i n t\left[\frac{2 \pi U}{h \omega}+1\right],
$$

$U$ is the effective ionization energy. This exceeds the ionization potential $U_{i}$ by a value equal to the mean kinetic energy of the free electron oscillating in the electromagnetic wave: ${ }^{6}$

$$
U=U_{i}+\frac{e^{2} E^{2}}{4 m \omega^{2}} .
$$

Using typical parameters for the ICA experiment and referring to Eq.(7), Eqs.(A.1)-(A.3) become

$$
\begin{gathered}
A=1.78 \times 10^{14} n^{3 / 2}(0.34 \times I)^{n}, \\
n=\operatorname{int}\left[\frac{25 U}{3}+1\right],
\end{gathered}
$$

and

$$
U=15.4+1.05 \times 10^{2} I
$$

where $I$ is in $T W / \mathrm{cm}^{2}$ and $U$ is in $\mathrm{eV}$.

For a short wavelength laser driver, multiphoton ionization starts to dominate the gas breakdown process and the gas will be fully ionized at a relatively low laser intensity. However, at the lower pressure typical for a near-resonance ICA scheme, ${ }^{13}$ even though the gas is completely ionized, reflection and attenuation of the laser beam will not happen due to $N_{m} Z \ll 10^{-3} N_{c r}$ (see Appendix B). Thus, gas breakdown would be no longer a limitation for ICA. 


\section{B. Gas Breakdown Threshold at Different ICA Conditions}

\begin{tabular}{cccc}
\hline \hline Parameters & $H_{2}$ & $H_{2}$ & $L i$ \\
\hline$U_{i}(\mathrm{eV})$ & 15.4 & 15.4 & 5.4 \\
$\lambda(\mu \mathrm{m})$ & 10.6 & 0.248 & 0.8 \\
$p(\mathrm{~atm})$ & 1.7 & 0.01 & 0.01 \\
$N_{c r}\left(\mathrm{~cm}^{-3}\right)$ & $1.0 \times 10^{19}$ & $1.8 \times 10^{22}$ & $1.7 \times 10^{21}$ \\
$N_{m}\left(\mathrm{~cm}^{-3}\right)$ & $4.5 \times 10^{19}$ & $2.8 \times 10^{17}$ & $2.8 \times 10^{17}$ \\
$\mathrm{DM}$ & Avalanche & Multiphoton & Multiphoton \\
$I_{t h}\left(T W / \mathrm{cm}^{-2}\right)$ & 10 & No Limit & No Limit \\
$P_{T}$ & $<10^{-6}$ & NA & NA \\
$P_{M}$ & NA & 1.0 & 1.0 \\
\hline \hline
\end{tabular}

where DM is dominant mechanism, NA is 'Not Applicable', $P_{T}$ and $P_{M}$ are the probabilities of tunneling and multiphoton ionization. 


\section{References}

1. J.R. Fontana and R.H. Pantell, J. Appl. Phys. 54, 4285 (1983).

2. W.D. Kimura, et al, Phys. Rew. Lett. 74(4), 546 (1995).

3. I.V. Pogorelsky, W.D. Kimura, C.H. Fisher, F. Kannari, and N.A. Kurnit, Approach to Compact Terawatt $\mathrm{CO}_{2}$ Laser System for Particle Acceleration, 6th Workshop on Advanced Accelerator Concepts, June 12-18, 1994, Lake Geneva, WI.

4. J.R. Fontana, W.D. Kimura, L.C. Steinhauer, and I.V. Pogorelsky, Proc. of Particle Accelerator Conference, May 17-20, 1993, Washington, DC., 2614.

5. V.L. Ginzburg, Propagation of Electromagnetic Waves in Plasma, Gordon \& Beach, NY, 1961.

6. L.V. Keldysh, Sov. Phys. JETP, 20, 1307(1965).

7. Yu.P. Raizer, Sov. Phys. USPEHY, 8, 650(1966).

8. P.B. Corkum, IEEE J. Quant. Electr., EQ-21, 216(1985)

9. L.S. Frost and A.V. Phelps, Phys. Rev., 127, 1621(1962).

10. A.G. Engelhardt and A.V. Phelps, Phys. Rev., 131, 2115(1963).

11. V.T. Platonenko and V.D. Taranukhin, Sov. J. Quant. Electr., 13, 1459 (1983).

12. I.V. Pogorelsky, J. Fischer, K. Kusche, M. Babzien, N.A. Kurnit; I.J. Bigio, R.F. Harrison, and T. Shimada, IEEE J. Quant. Electr., EQ-31, 556 (1995).

13. L.C. Steinhauer and W.D. Kimura, J. Appl. Phys., 68, 4929 (1990).

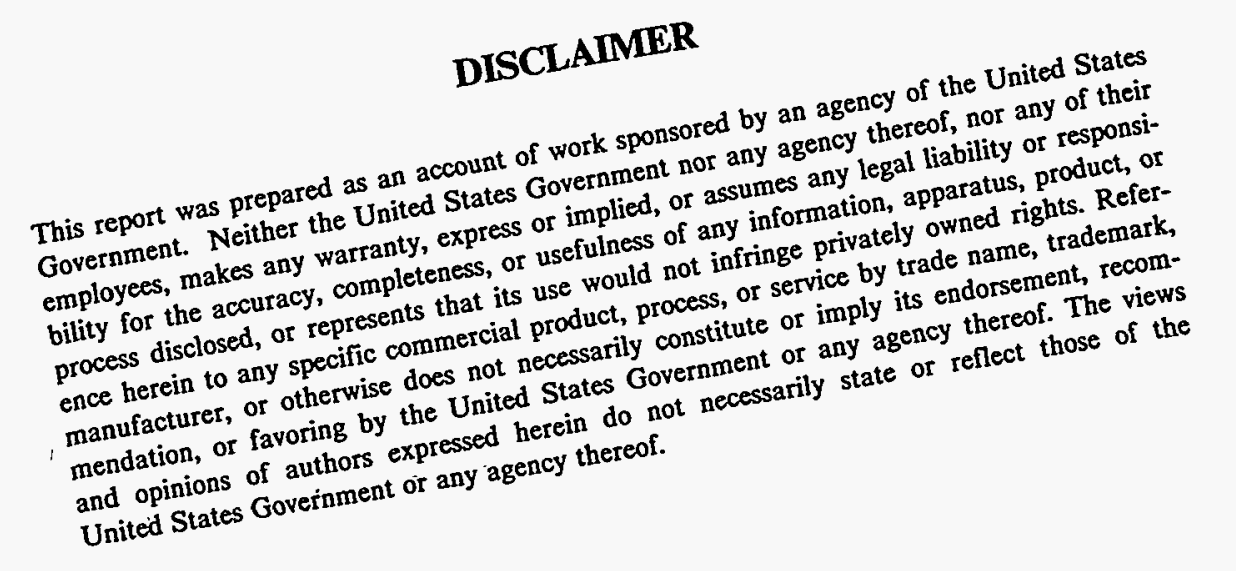

